\title{
Postpartum Depression (PPD)
}

\author{
Beth Buxton, LCSW
}

Massachusetts Department of Public Health beth.buxton@state.ma.us 


\section{Mental Health Disorders}

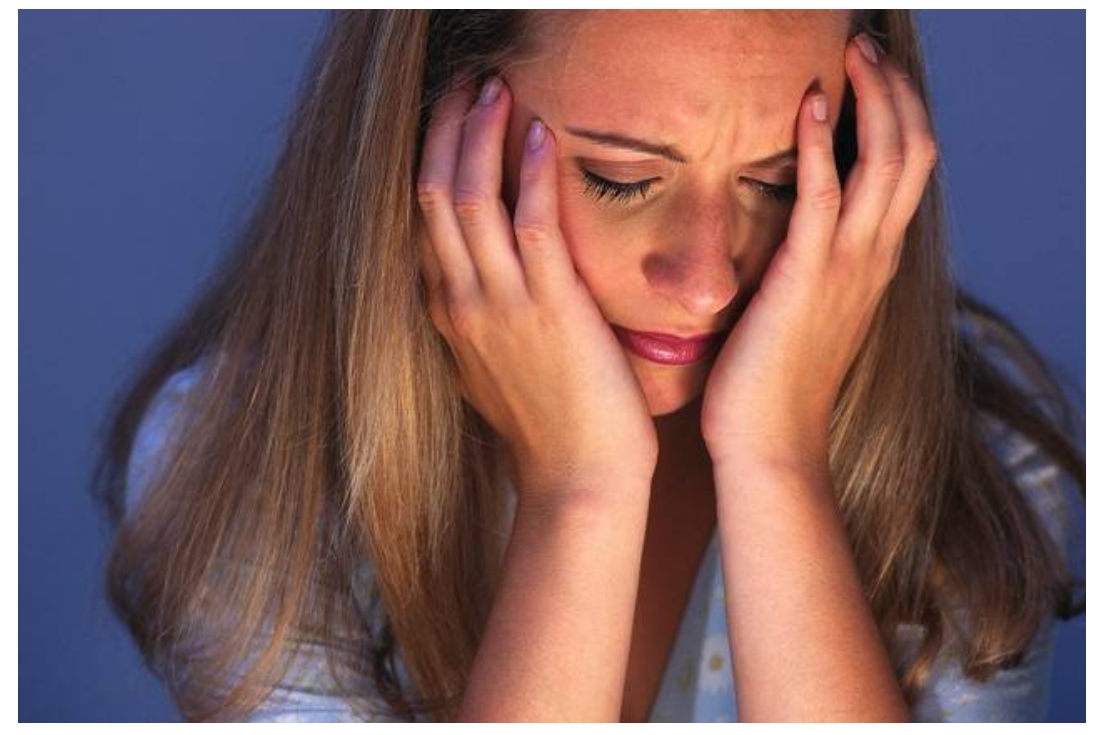

- An estimated 57.7 million adults $(26.2 \%$ of adult population) suffer from a diagnosable mental health disorder in the US in any given year

- The leading cause of disability for $15-44$ year old population in US.

- Affects women twice as much as men 


\section{Maternal Mental Health}

- Incidence of Depression is greatest during the reproductive years

- Maternal Mental Health or Maternal Depression is a multifaceted illness that describes a range of physical and emotional changes that many mothers experience during pregnancy or

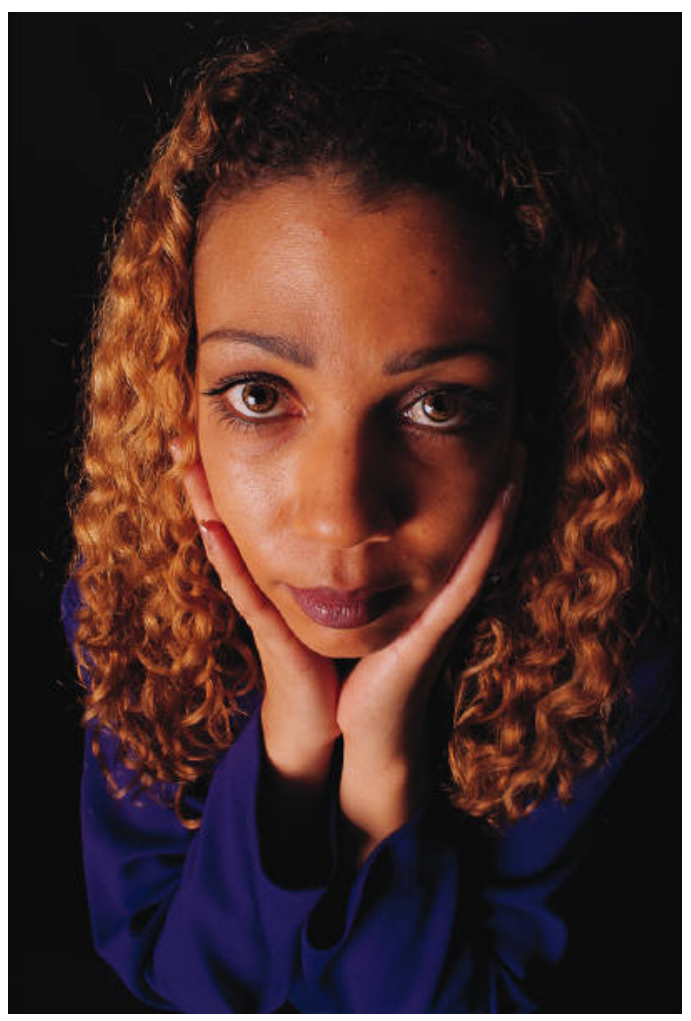
postpartum. 


\section{Factors that Increase Risk}

- History of a mental health disorder including postpartum depression

- Severe premenstrual syndrome

- Family history of depression

- Recent/recurrent stressful events such as

- Poverty

- Interpersonal violence

- Lack of social supports 


\section{General Signs \& Symptoms}

- Feeling restless

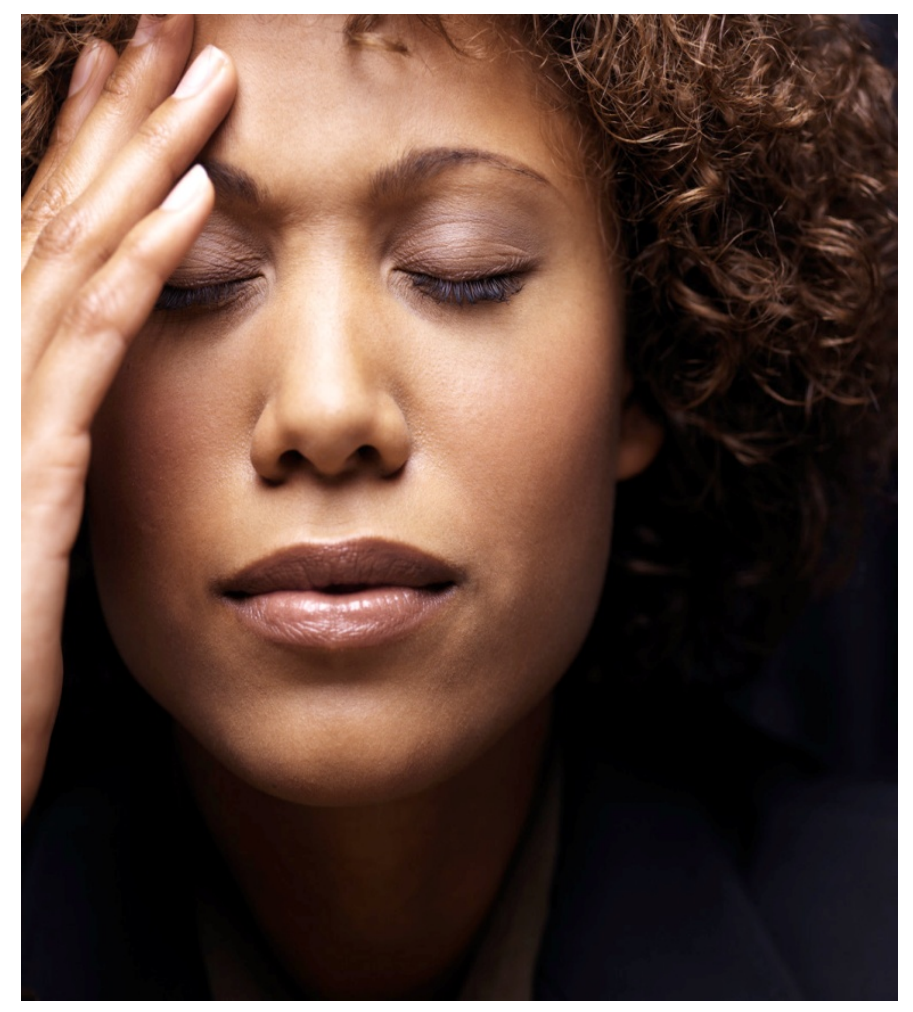

- Increased crying/crying often

- Lack of energy

- Feeling anxious or jumpy

- Feeling irritable or angry

- Sleeping too much/not enough

- Eating too much/not enough

- Headaches; chest pains

- General aches and pains

- Loss of interest in family

- Feeling guilt and despair

- Afraid of hurting oneself/infant

- Hyper-vigilance 


\section{Postpartum Blues}

- Also known as the "Baby Blues"

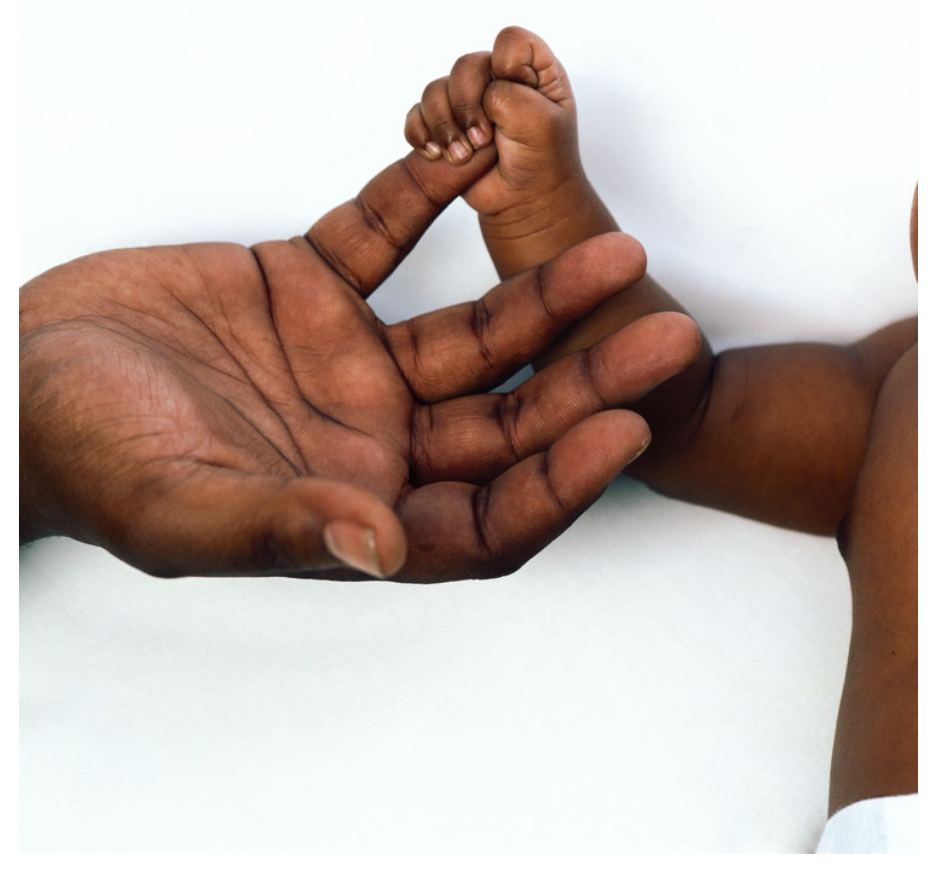

- 50\%-80\% of all new mother experience within the first 10 days following birth

- Symptoms usually mild and do not interfere with functioning or infant care giving 


\section{Postpartum Depression}

- $10 \%-20 \%$ of all new mothers experience this significant, clinical condition

- Mother experiences major depressive episodes

- Lasting two weeks to two years after birth 


\section{Postpartum Psychosis}

- Rare; between 1 - 4 women out of 1,000 births will experience this psychiatric emergency

- Usually develops within one week after birth

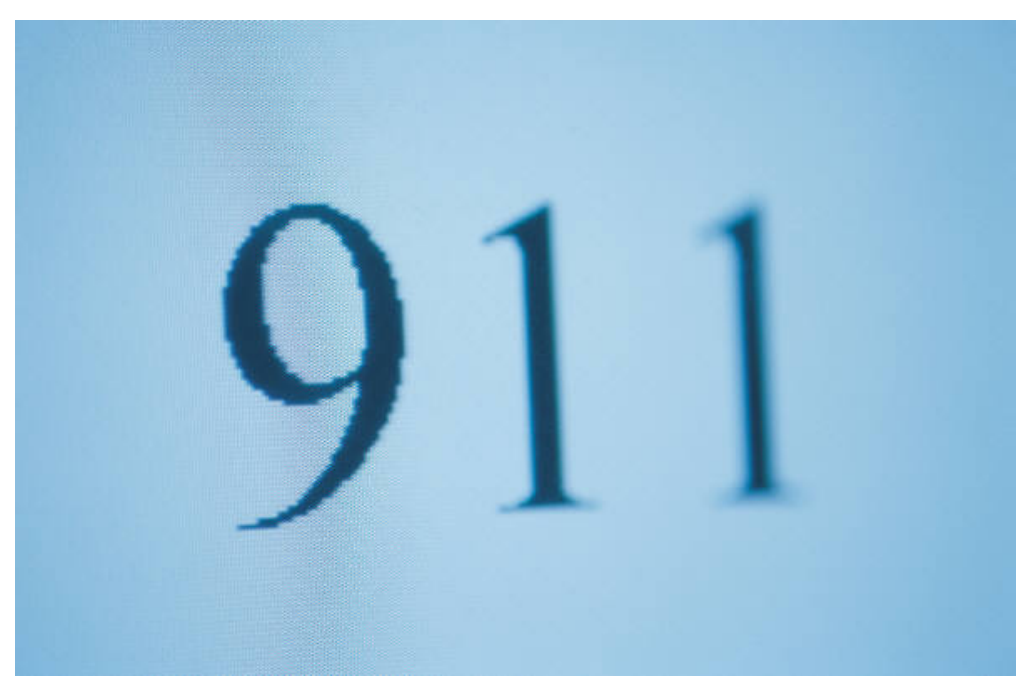

- Symptoms include hallucinations, paranoia, suicidal and homicidal ideation 


\section{MDPH Definition of PPD}

- The term postpartum depression describes the range of physical, emotional, and behavioral changes that many new mothers experience following the delivery of their babies, with symptoms most commonly starting 1-3 weeks after delivery.

- Women with postpartum depression have such strong feelings of sadness, anxiety or despair that they have trouble coping with their daily tasks. Symptoms of this condition can range from mild to severe. About $80 \%$ of new mothers have depressed mood known as the 'baby blues" that resolves within 2 weeks without treatment.

- Postpartum depression is a much more serious condition which can impact the health of the infant and the mother and requires treatment. In some cases, new mothers may have postpartum psychosis, a relatively rare psychiatric emergency. (citation: Dell D. (2008) Clinical Updates in Women's Health Care: Mood and Anxiety Disorders) 


\section{PPD can be difficult to identify}

- Symptoms confused with normal postpartum adaptation "baby blues"

- Moms do not know what to expect

- Moms do not want providers to think they are bad mothers 


\section{Maternal Impact}

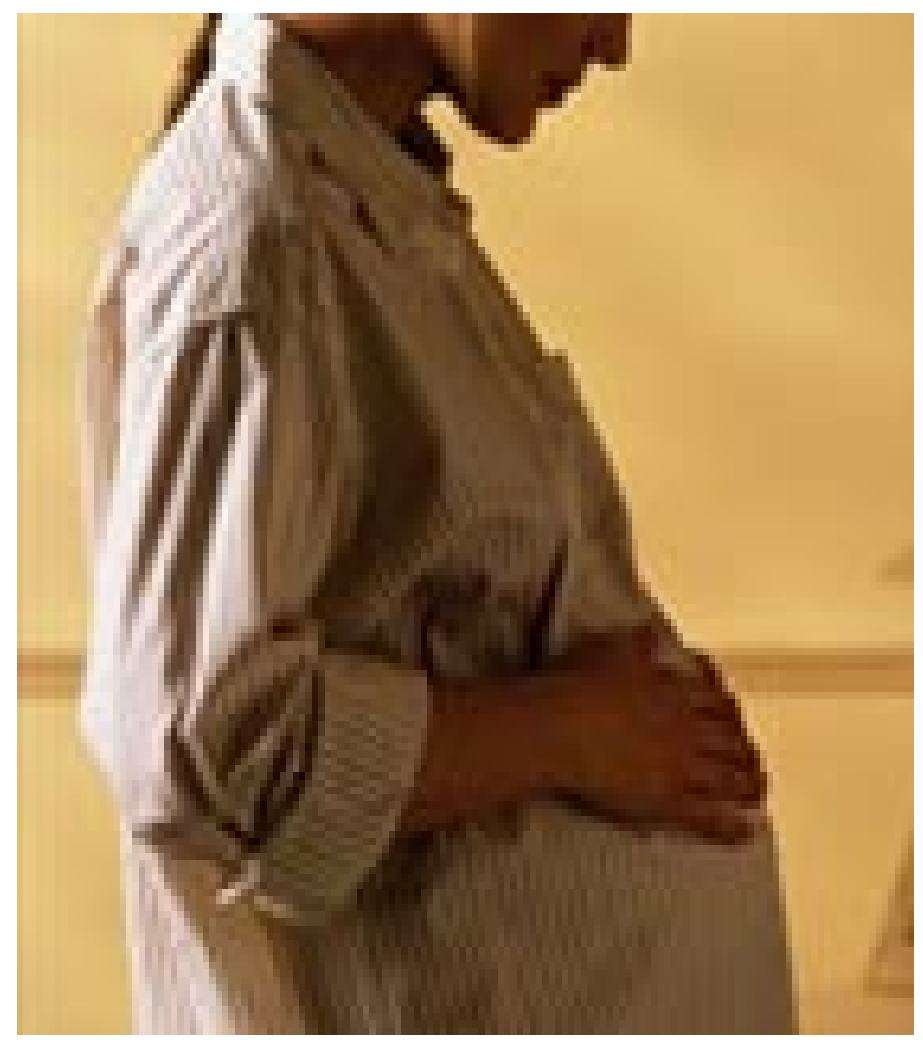

- Increased maternal substance abuse

- Co-morbidity with interpersonal violence

- Compromised parenting

- Reduced maternalinfant attachment 


\section{Impact on Newborn}

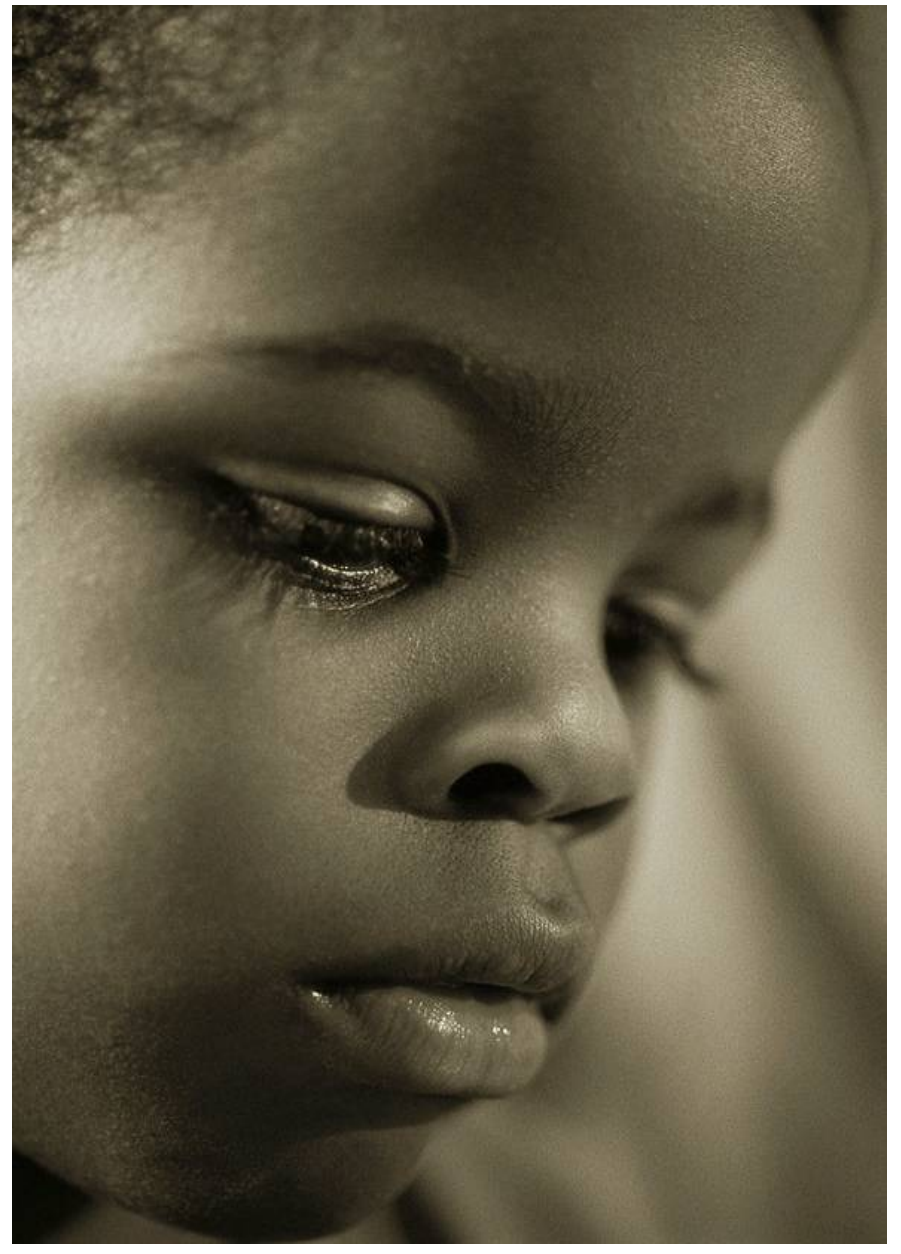

- Reduced attachment

- Higher stress levels

- Effects brain development

- Jeopardizes infant language, physical, intellectual, and emotional development.

- Higher prevalence of behavior disorders in older children 


\section{Family Voices}

MNPI Digital Stories

http://www.mass.gov/dph/newparents 


\section{PPD is Treatable}

- Highly treatable with short term Cognitive/Behavioral Therapy

- Interpersonal therapy effective for mild and moderate PPD

- More severe depression may require antidepressants (Selective Serotonin Reuptake Inhibitors \{SSRIs\} most commonly used) 


\section{What can providers do?}

Identification and Universal Screening

- Avoid assumptions - looks can be deceiving

- Assure mother that concerns about PPD do not make her an unhappy or bad mother.

- Normalizing - Becoming a new parent can be both overwhelming and wonderful - it is normal for a new family to experience both emotions. 


\section{How screening tools help}

- Screening improves detection and identification of prenatal depression and postpartum depression

- Help predict future depression

- Can track depression throughout the year

- Can be used as an opening for discussion around maternal and family issues 


\section{PPD Screening Tools}

Patient Health Questionnaire: Scaled PHQ-2

1. Answer key for 2 questions below

- Not at all: 0

- Several days: 1

- More than half the days: 2

- Nearly every day: 3

2. In past 2 weeks, how often have you been bothered by:

- Little interest or pleasure in doing things?

- Feeling down, depressed or hopeless?

Positive if 3 or more points

Efficacy Test Sensitivity @ 83\% and Test Specificity @ 92\% 


\section{PPD Screening Tools}

- Edinburgh Postnatal Depression Scale (EPDS)

- Self-administered

- 10 questions

- Available in multiple languages

- The validation study showed that mothers who scored above a threshold 12/13 were likely to be suffering from a depressive illness of varying severity 


\section{Massachusetts Legislation}

- An Act Relative to Postpartum Depression (PPD): Chapter 313 of the Acts of 2010 was signed into law by Deval Patrick on August 19, 2010.

- Law authorizes the DPH to develop a culture of awareness, de-stigmatization, and screening for perinatal

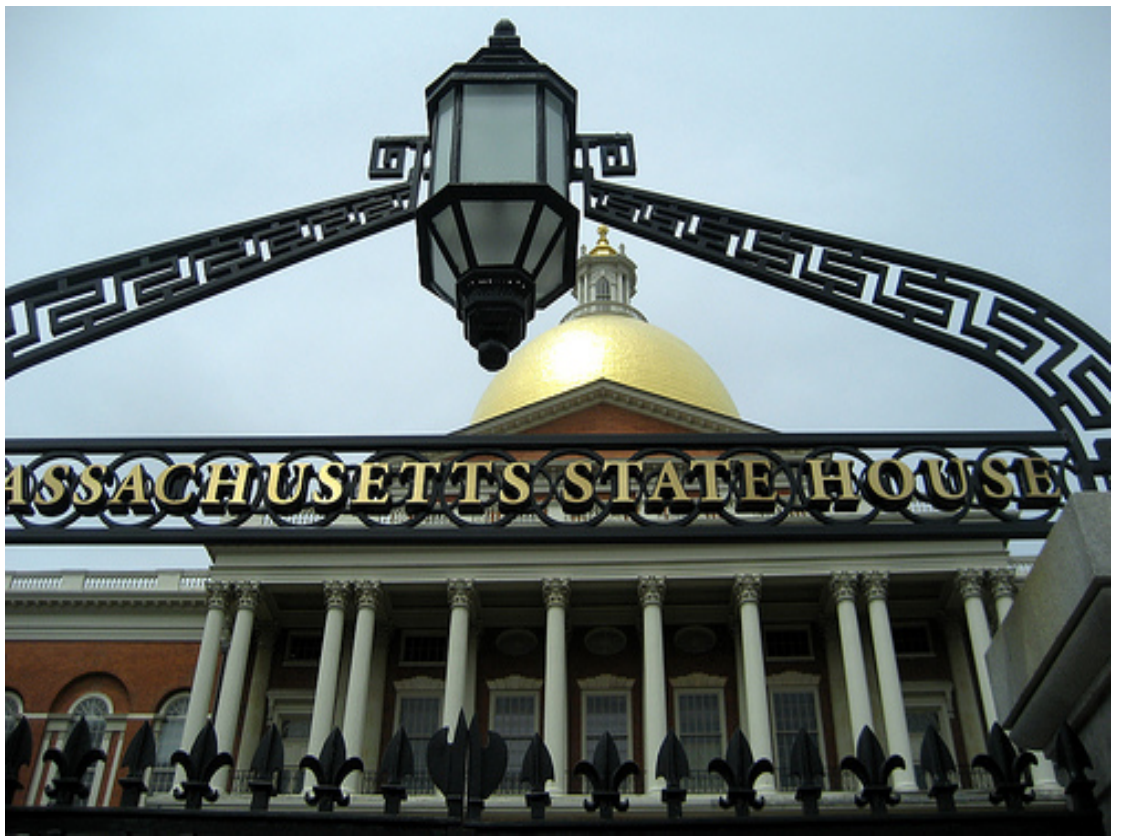
depression 


\section{Resources Developed}

- Standards for Effective PPD screening

- Recommendations for health care providers and health plans for reporting PPD screening data

- PPD Screening Tool Grid

- PPD Resource List \& Bibliography

- PPD Web Page on MDPH Web Site:

http://www.mass.gov/eohhs/gov/dep

Standards for Effective Postpartum Depression Screening in Massachusetts

Recommendations for Health Plans and Health Care Providers Postpartum Depression (PPD) Screening Data Reporting

Massachusetts Department of Public Health January 2012 artments/dph/programs/familyhealth/postpartum-depression 


\section{MA Regionally Based Coalitions \& Task Forces}

- Cape \& the Islands

- http://www.capecoalition.com/mdtf.html

- Western Mass (based in Amherst)

- MotherWoman: www.motherwoman.org

- North Shore

- North Shore Postpartum Depression Task Force: http://northshorepostpartumhelp.org

- South Shore

- The South Shore Postpartum Support Network: www.southshorepostpartum.org

- Postpartum Support International of MA:

- www.postpartumma.org 


\section{Massachusetts \\ Health Promotion Clearinghouse}

- http://maclearinghouse.com

- or call 800-952-6637 for free brochures
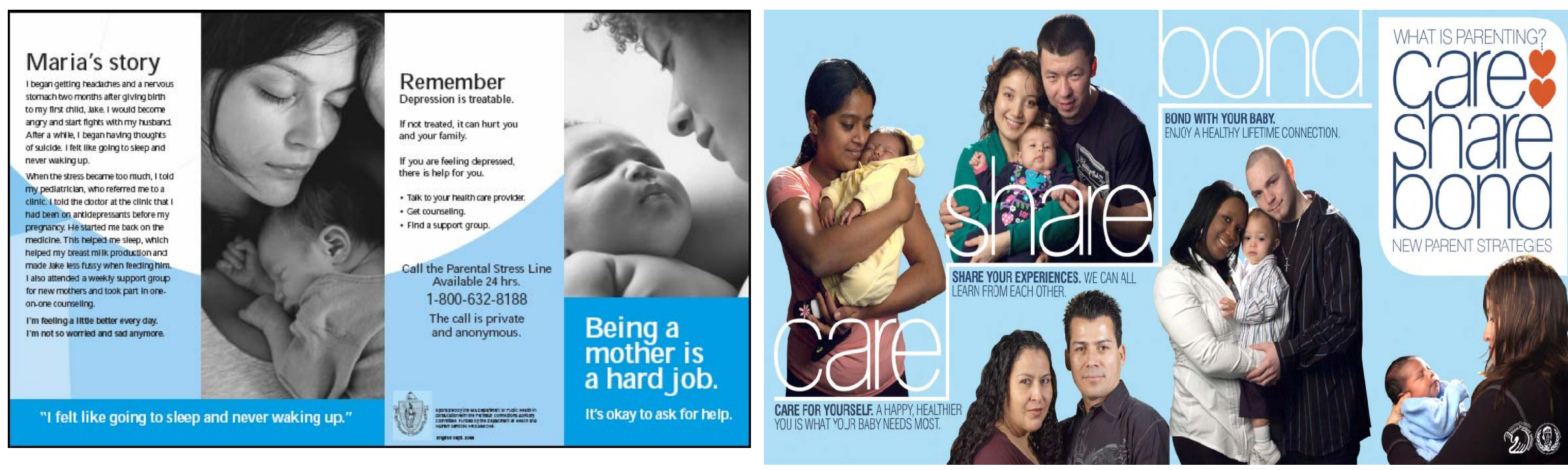